\title{
A RELEVÂNCIA DA MONITORIA PARA OS MONITORES E MONITORADOS DO CEFET-MG CAMPUS ARAXÁ
}

DOI: 10.37702/2175-957X.COBENGE.2021.3392

bruno patto graciano natal - bruno.natal02@gmail.com

Centro Federal de Educação Tecnológica de Minas Gerais

rua almeida campos 11

38183-222 - araxá - MG

Guilherme De Lima Resende Martins - guilhermelresende@gmail.com entro Federal de Educação Tecnológica de Minas Gerais

Rua Paulo De Souza 155

38180-281 - Araxá - MG

Kleber Lopes Fontoura - kleberfontoura@gmail.com entro Federal de Educação Tecnológica de Minas Gerais Alameda do Sol 320 38181-415 - Araxá - MG

Leonardo de Oliveira Souza Mendonça - leonardo.somendonca@gmail.com entro Federal de Educação Tecnológica de Minas Gerais Rua Antônio Ribeiro da Silva 53 38170-000 - Perdizes - MG

Marcos Vinícius Alves Pereira - mvapereira97@gmail.com entro Federal de Educação Tecnológica de Minas Gerais

Resumo: A monitoria é de suma importância na vida acadêmica do corpo discente, pois agrega positivamente em seu currículo, além de aperfeiçoar suas habilidades ao longo da formação. Incumbe ao monitor auxiliar o educando em suas fragilidades referentes às matérias lecionadas. Dessa forma, o presente artigo objetiva analisar a forma como a monitoria é utilizada para potencializar a aprendizagem colaborativa e autorregulada dos estudantes universitários, com a finalidade de propor intervenções que possam melhorar o programa de monitoria acadêmica da Universidade e a experiência pedagógica dos alunos-monitores, 
professores-orientadores e educandos. Para isso, foram aplicados dois formulários para os alunos dos cursos de Engenharia de Automação Industrial e Engenharia de Minas do CEFET-Araxá. O primeiro formulário visava entender os benefícios e dificuldades dos monitores e o outro tinha como intuito principal ouvir os alunos que compareciam assiduamente às monitorias. Com os dados obtidos através das duas pesquisas, foi analisado os pontos positivos e negativos da monitoria e o que ela impactou, especialmente na formação do corpo discente. Foi possível observar que para os educandos e monitores, a monitoria trouxe melhoras em sua capacidade de comunicação, aumento em suas notas, além de um foco maior nos estudos. Contudo, é possível também notar alguns problemas que dificultam a instrução da monitoria, como falta de espaço adequado para o programa e a presença de alunos desnivelados quanto ao conteúdo reforçado.

Palavras-chave: Monitor. Monitoria. Benefícios. Dificuldades. Formação. 


\section{INTRODUÇÃO}

O exercício da Engenharia requer, primeiramente, fundamentação teórica, técnica e científica e um alicerce em princípios éticos sólidos. Apesar disso, é possível observar um número cada vez maior de acadêmicos com dificuldade em atingir um currículo escolar adequado e desenvolver habilidades e competências demandadas pelo mercado de trabalho contemporâneo.

De acordo com Beltran, são atribuições das Instituições de Ensino Superior (IES) proporcionar ao aluno um processo de aprendizado ativo, cognitivo, construtivo, significativo, mediado e autorregulado. Dessa forma, as IES têm papel fundamental na formação do discente em sua integralidade, não sendo, portanto, somente um adicionador de conteúdos técnicos e científicos. Para isso, tem sido implementado um esforço cada vez mais significativo das IES em promover atividades extracurriculares educativas e pedagógicas, visando a capacitação do acadêmico e aperfeiçoamento de suas habilidades teóricas e técnicas.

Segundo Tavares, faz-se necessário ampliar, com urgência, as ferramentas pedagógicas das IES, sendo o investimento no âmbito da docência uma alternativa excelente. É possível observar um investimento das IES em tais estratégias e práticas que facilitam o aprendizado e o processo pedagógico. Tal investimento é incentivado pelo Ministério da Educação (MEC), por conferir bonificações às IES que adotam atividades extracurriculares. Uma maior nota pelo MEC confere maior prestígio social e reconhecimento mercadológico.

Neste contexto, ganha relevância a Monitoria Acadêmica (MA), entendida como mais uma maneira de apoio ao educando que consiste em um auxílio alternativo dado pelo discente-monitor ao monitorando, por meio de um amparo às fragilidades inerentes à matéria em questão, revisões extras e ajuda com a rotina de estudos. Além disso, cabe ao monitor reforçar os conhecimentos lecionados em aula pelo professor, observar as aspirações, expectativas e dificuldades dos alunos e favorecer a integração entre a teoria e a prática, quando possível.

Monereo comenta que nem sempre a maneira como o professor explica o conteúdo é claramente entendida por todos alunos, sendo praticamente impossível que o docente saiba o que se passa na cabeça daqueles que o escutam. Nesse sentido, a MA surge como uma excelente proposta de integração entre o aluno e o professor, ao proporcionar um modelo de aprendizado relacional e totalmente interativo, em que o aluno monitor colabora com o professor elucidando as dificuldades da sala e os pontos que devem ser melhor trabalhados.

Dessa forma, o presente artigo objetiva analisar, por meio de um questionário aplicado aos alunos monitores do curso de Engenharia de Automação Industrial e Engenharia de Minas do CEFET-Araxá, a forma como a monitoria é utilizada para potencializar a aprendizagem colaborativa e autorregulada dos estudantes universitários, com a finalidade de propor intervenções que possam melhor o programa de MA da Universidade e a experiência pedagógica dos alunos monitores e professores orientadores. 


\section{MATERIAIS E MÉTODOS}

Para a geração dos resultados acerca do estudo proposto neste artigo, fez-se necessário a aplicação de dois formulários, através da plataforma Google Forms, criandose perguntas de múltipla escolha e uma pergunta discursiva. O primeiro formulário, destinado à pesquisa com os monitores da graduação, foi dividido em duas seções. $\mathrm{Na}$ primeira seção, realizou-se perguntas pessoais afim de se conhecer melhor o perfil dos monitores e colher informações importantes sobre eles. Já na segunda seção, foram feitas perguntas específicas relacionadas ao tempo em que a monitoria foi aplicada, o que permitiu avaliar a influência da MA para os monitores, tanto no âmbito acadêmico, como no profissional.

O segundo formulário, foi destinado aos alunos da graduação que frequentaram assiduamente as monitorias ofertadas. Tal formulário teve a proposta de avaliar o impacto da participação rotineira na MA para os alunos, visando avaliar se tal método de ensino é produtivo e eficiente.

Depois de finalizada a elaboração dos formulários, com o auxílio do Departamento de Formação Geral do Centro Federal de Educação Tecnológica de Minas Gerais (CEFET$\mathrm{MG}$ ), Unidade de Araxá, obteve-se os dados dos monitores dos dois últimos anos, que representam uma amostra desta classe. Dessa forma, foi possível entrar em contato e aplicar o formulário aos monitores. No total, obteve-se 7 avaliações diferentes. Além disso, foi solicitado aos os alunos da graduação que respondessem o formulário proposto a eles, obtendo-se 19 avaliações. Com os resultados em mãos, foi possível analisar e dissertar de forma crítica sobre a atual situação da monitoria na instituição, verificando seus pontos positivos e negativos, principalmente para os discentes.

\section{DISCUSSÃO}

A importância da monitoria no ensino superior extrapola a mera obtenção de um currículo acadêmico interessante, que privilegia a aprovação de discentes em programas de mestrado, intercâmbios e até mesmo estágio. Além desses benefícios, a MA contribui no processo de aprendizado do aluno monitorando, do monitor e ajuda o professor em seu processo pedagógico.

\subsection{Condição da IES para a atuação dos monitores}

Ao iniciar a análise dos resultados, ressalta-se que houve, no total, no CEFET-MG campus Araxá, nos dois anos anteriores à pesquisa, 14 monitores na Formação Geral. Destes, apenas 7 responderam ao questionário aplicado conforme elucidado em Materiais e Métodos.

Ressalta-se que na IES analisada, os estudantes devem atender aos pré-requisitos contidos no edital (2020): ter obtido, na matéria que pretendem ser monitores, média mínima de 60 pontos, ter cursado a disciplina que o aluno pretende ser monitor e disponibilizar 20 horas semanais para o desempenho das funções de monitor.

A média no edital anterior (2019), para as matérias da formação geral, era de 65 pontos, como mostrado na Tabela 1. O motivo de tal mudança foi a baixa adesão ao processo seletivo de MA, uma vez que os alunos interessados não atingiam a pontuação exigida. Dessa forma, como uma maneira de não prejudicar os demais discentes pela 
ausência de monitores, o pré-requisito média foi facilitado, o que aumentou o número de inscrições e monitores na formação geral, apesar da adesão ainda ser baixa.

Tabela 1: média mínima exigida na matéria em que o discente pretende ser monitor conforme o ano do edital.

\begin{tabular}{|c|c|}
\hline Ano analisado & Média mínima exigida \\
\hline 2018 & $70 \%$ \\
\hline 2019 & $65 \%$ \\
\hline 2020 & $60 \%$ \\
\hline
\end{tabular}

Fonte: Própria

Ao se inscreverem para o processo seletivo da MA, os alunos ficam cientes que deverão ser submetidos à uma prova teórica com o conteúdo integral da matéria escolhida e uma entrevista com o professor orientador. O monitor, ao ser aprovado no processo seletivo, obtendo nota suficiente na avaliação e bom desempenho na entrevista, recebe uma bolsa no valor de 350 reais (o bolsista não poderá receber bolsa paga por instituições públicas ou privadas, incluindo remunerações referentes à estágios, excluindo bolsa de assistência estudantil, nem possuir vínculo empregatício de qualquer natureza). Ao final de cada mês, o aluno monitor deve apresentar um relatório à formação geral para que a bolsa seja debitada.

Conforme o edital, os objetivos da MA no CEFET-Araxá são: contribuir para o desenvolvimento de aptidões docentes do aluno; contribuir para a formação acadêmica do aluno; possibilitar o compartilhamento de conhecimentos adquiridos com outros alunos; promover a cooperação entre os corpos discentes e docente para a melhoria do ensino e contribuir para minimizar os problemas de repetência, evasão e de falta de motivação dos alunos.

\subsection{Características dos monitores do CEFET-Araxá}

O presente estudo teve como objetivo analisar a efetividade da MA da formação geral do CEFET-MG campus Araxá nos últimos 2 anos por meio da aplicação de um formulário contendo perguntas abertas e fechadas. Neste período analisado, houve 14 monitores, porém apenas 7 respondeu ao questionário (1 aluno do curso de Engenharia de Automação Industrial e 6 alunos da Engenharia de Minas).

Dos alunos entrevistados, 2 estão matriculados entre o $5^{\circ}$ e $7^{\circ}$ período, 2 entre o $8^{\circ}$ e $10^{\circ}$ período e 3 já formados. O Gráfico 1 demonstra as matérias que foram contempladas pelos monitores.

Gráfico 1 - matérias da formação geral e porcentagem de monitores de acordo com os entrevistados. 


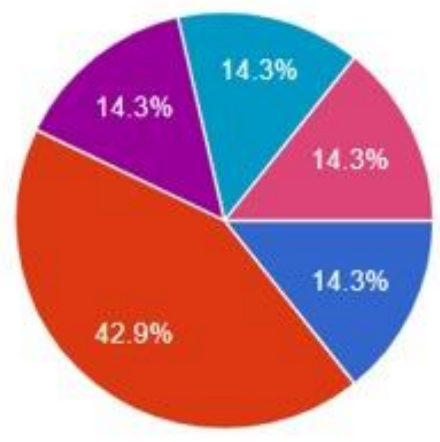

Geometria Analitica e Álgebra Vetorial

Resistência dos materiais

Hidráulica e física

Fonte - Própria

\section{Araxá}

\subsection{Sugestões dos monitores para melhorar o programa de MA do CEFET-}

Em uma pergunta aberta, demonstrada na Tabela 2, feita aos monitores, foi questionado "Em sua opinião, o que você acha que deveria mudar na monitoria? ". Dentre as respostas, 6 alunos, de 7 no total, citaram o espaço físico para a monitoria como um importante fator de melhora.

Tabela 2: opinião dos monitores sobre possíveis mudanças que melhorariam o programa de MA.

\begin{tabular}{|c|}
\hline O que você acha que deveria mudar na monitoria? \\
\hline "Salas melhores." \\
\hline "Os alunos deveriam ser mais incentivados a participar, o número de alunos indo às \\
monitorias não era tão grande." \\
\hline $\begin{array}{c}\text { "Acho que deveria haver um espaço e materiais adequados, tais como quadros, mesas e } \\
\text { cadeiras, para que os monitorados possam absorver o conteúdo em um local confortável e } \\
\text { aprazível, o que, consequentemente,, melhora o processo de aprendizado, tornando-o mais } \\
\text { agradável." }\end{array}$ \\
\hline "Ter uma sala maior e própria para monitoria." \\
$\begin{array}{c}\text { "Implantação de um espaço próprio para a monitoria. Também, uma organização melhor na } \\
\text { forma que os monitores lecionam. Às vezes, os monitores se preparam para a monitoria e } \\
\text { ninguém comparece, o que compromete o cumprimento da horas semanais mínimas." }\end{array}$ \\
\hline
\end{tabular}

Fonte: Própria

\subsection{Motivos pelos quais os monitores do CEFET-Araxá se inscrevem no processo seletivo de MA}

Definir os motivos pelos quais os monitores da IES pesquisada se inscrevem no processo seletivo de MA é fundamental para conhecer o perfil do aluno da instituição e intervir de forma positiva com a finalidade de melhorar o programa de monitoria da Universidade.

Quando questionados sobre o motivo pelos quais o aluno optou por se inscrever no processo de monitoria, $71,4 \%$ dos alunos responderam que a principal motivação é a bolsa ofertada ao aluno monitor. 


\subsection{Melhorias que a MA proporcionou ao aluno monitor}

A MA tem como objetivo, além de contribuir para a melhoria da qualidade de ensino, educação e extensão, contribuir para a melhoria do processo de aprendizagem dos alunos, do professor-orientador e do aluno-monitor. Os ganhos para o monitor, geralmente, vêm em forma de notas melhores, ampliação do currículo acadêmico e conhecimento de uma nova possibilidade de trabalho: a área acadêmica.

Foi questionado aos entrevistados "Em quais aspectos a monitoria te agregou?", pergunta em que podia-se marcar mais de uma opção. 5 alunos marcaram a opção "Melhora na Comunicação", sendo um total de $71,4 \%$ dos entrevistados. As outras opções, tais como: melhora em notas, networking profissional e renda obtiveram uma marcação cada. Além disso, 2 alunos marcaram que a monitoria permitiu dedicação exclusiva aos estudos, muito provavelmente pela bolsa que esta oferece, o que isenta financeiramente $o$ aluno de procurar trabalhos ou estágios remunerados. Em relação à Bolsa de Monitor, questionou-se se esta era satisfatória. $57,1 \%$ a considerou ótima, $28,6 \%$ satisfatória e 14,3 $\%$ ruim.

\subsection{Dificuldades dos monitores}

Os alunos-monitores foram questionados sobre as maiores dificuldades impostas pela monitoria, conforme o Gráfico 2 abaixo. 100\% dos alunos apontaram o espaço físico inadequado como uma grande dificultador do processo de MA. Além disso, 2 alunos citaram que o fato de haver alunos desnivelados quanto ao conteúdo também dificultou o processo.

Gráfico 2: Qual foi a sua maior dificuldade como monitor?

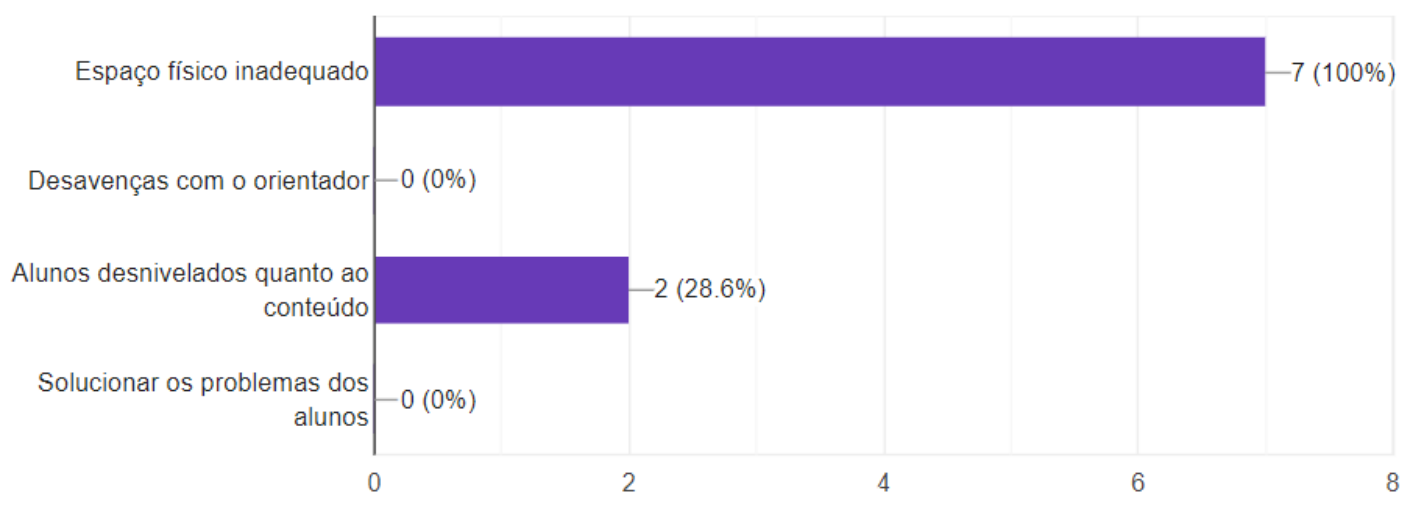

Fonte: Própria

\subsection{Desempenho acadêmico}

Sabe-se que o objetivo da MA, em muitas IES, é promover uma melhora no desempenho acadêmico dos cursos de graduação, além de contribuir para o processo de formação discente e incentivar o monitor o interesse pela carreira acadêmica.

Em relação ao desempenho acadêmico do monitor durante ou após o período de MA, foi perguntado se houve melhora, piora ou se este se manteve o mesmo. $57,1 \%$ dos 
alunos responderam que houve melhora no desempenho, enquanto $42,9 \%$ afirmou que 0 desempenho acadêmico se manteve neutro.

\subsection{MA e auxílio no mercado de trabalho}

É evidente que o resultado da participação em MA denota ao monitor um avanço significativo e de qualidade em sua formação, uma vez que o aluno conhece, de perto, o trabalho do docente universitário, além de alcançar um número de créditos acadêmicos significativos ao seu currículo.

Santos; Lins (2007), acredita que a MA não somente auxilia o aluno cujo intuito após a Universidade é a área acadêmica, ela também é considerada uma oportunidade excelente na formação de profissionais que desejam se inserir no Mercado de Trabalho, visto que esta atividade tende à contextualizar a produção científica e o exercício profissional.

Perguntou-se aos monitores "Em quais aspectos você acha que a monitoria irá te ajudar no mercado de trabalho, ao se deparar com as adversidades impostas na sua profissão? ". 6 alunos responderam que o auxílio se daria por meio de uma melhor capacidade de comunicação adquirida na MA. 1 aluno respondeu que a MA o ajudaria ao permitir que ele encontrasse meios para solucionar problemas cotidianos do trabalho com mais facilidade. 3 alunos citaram o fato de lidar melhor com superiores hierárquicos um facilitador no mercado de trabalho.

\subsection{Apoio do professor orientador}

Nunes (2007), elucida em seu texto que a MA é representada por um espaço cujo intuito principal é a formação do aluno monitor, além do próprio professor orientador, bem como uma atividade extracurricular que visa contribuir para a melhoria da qualidade da educação. Neste contexto, o professor orientador é extremamente importante e tem como objetivo procurar envolver o monitor, ajudando-o nas fases de planejamento, interação em sala de aula, laboratório e na avaliação dos alunos e das aulas.

É importante ressaltar que a interação entre o monitor e seu professor-orientador deve ser aberta, clara e objetiva. Este diálogo aberto tende a favorecer e enriquecer 0 trabalho de preparação da disciplina, bem como as monitorias.

Ao serem questionados sobre o auxílio dos professores orientadores, $100 \%$ dos monitores respondeu que houve apoio, além de $100 \%$ classificar o professor orientador como ótimo, em uma escala que continha as opções ótimo, satisfatório, ruim e frustrante.

\subsection{Contribuições da MA para os alunos monitorados}

Para tentar elucidar a relevância da MA para os alunos aplicados, foi feito um questionário, conforme a tabela 3. No total, 19 alunos que participavam assiduamente das monitorias responderam às perguntas propostas. A partir da análise dos dados, é possível observar que a maioria dos discentes estudou e se envolveu mais nos estudos após a monitoria, faltou menos as aulas, aproveitou mais o tempo disponível de estudo e dedicou mais fora da monitoria. No entanto, apesar de a maioria responder que evitou os distratores, este ainda permanece sendo um problema quando comparado às demais respostas.

Tabela 3: Mudanças reveladas nos alunos monitorados durante o percurso da monitoria. Fonte: Frison (2012).

\begin{tabular}{|c|c|c|c|}
\hline Indicadores & Sim & Não & Total \\
\hline Faltei menos aulas & 17 & 2 & 19 \\
\hline Fui mais pontual com meus compromissos & 14 & 5 & 19 \\
\hline
\end{tabular}


28 a 30 de SETEMBRO

\begin{tabular}{|c|c|c|c|}
\hline Estive mais motivado, com mais vontade de estudar & 18 & 1 & 19 \\
\hline Aproveitei mais o meu tempo de estudo & 17 & 2 & 19 \\
\hline Estudei mais & 15 & 4 & 19 \\
\hline $\begin{array}{c}\text { Dediquei-me, fora da monitoria, para entender mais o assunto } \\
\text { Aprendi muito mais nas disciplinas que tinham monitoria em } \\
\text { comparação às que não tinham }\end{array}$ & 17 & 2 & 19 \\
\hline Tentei evitar os distratores (redes sociais, etc.) & 12 & 7 & 19 \\
\hline
\end{tabular}

Fonte: Própria

\section{CONSIDERAÇÕES FINAIS}

Entende-se que, as universidades que oferecem atividades extracurriculares são bonificadas pelo MEC (Ministério da Educação) e isso lhes proporciona uma melhor nota junto ao mesmo, assim como, maior prestigio social e reconhecimento mercadológico. Além disso, confere-se uma maior quantidade de alunos dispostos a estudar nestas universidades.

Dado os fatos a cima, torna-se evidente os benefícios diretos que a monitoria exerce sobre os monitorandos, tal como é demonstrado no questionário respondido por estes. Há uma melhora visível nos aspectos tangentes a vida acadêmica do aluno, como, melhoramento de notas, maior presença em aulas e uma potencialização da motivação para estudar - estes que são pontos de extrema relevância para a permanência do aluno na universidade.

Após a análise dos dados coletados, por meio do questionário destinado aos monitores, é possível compreender como a monitoria os afeta de forma majoritariamente positiva. Sendo assim, destacam-se pontos como: melhora na capacidade de comunicação e desempenho acadêmico; no tocante, o auxílio promovido a estes sujeitos no ingresso no mercado de trabalho: a melhora na capacidade de comunicação é apontada como sendo de grande valia para os monitores, assim como, a facilidade de lidar com superiores hierárquicos.

Tendo em vista as respostas obtidas pelos questionários, podemos demonstrar como a monitoria afeta não somente o monitor, mas também o monitorando, assim como a própria universidade. Faz-se necessário a atenção para com aspectos fundamentais a serem melhorados, com o intuito de fomentar a atividade de monitoria, como, melhores acomodações, dadas as condições precárias enfrentadas pelos monitores na universidade em questão; redução na nota de corte exigida para ingresso na monitoria, que por sua vez, foi em parte responsável pelo ingresso de uma quantidade menor de monitores em anos anteriores. A monitoria se mostra como uma atividade carregada de benefícios para com todos os envolvidos, fortificando aspectos acadêmicos e sociais de indivíduos, possibilitando a formação de melhores profissionais e cidadãos.

\section{REFERÊNCIAS}

BATISTA, J. B., \& FRISON, L. M. B. F. Monitoria e aprendizagem colaborativa e autorregulada. In: D. Voos, \& J. B. Batista (Orgs.). Sphaera: sobre o ensino de matemática e de ciências, Porto Alegre: Premier, p. 232-247, 2009. 
MONEREO, C. Aprender entre iguais e com iguais. In: D. Duran, \& V. Vidal (Orgs.).

Tutoria: aprendizagem entre iguais. Porto Alegre: Artmed, 2003.

NUNES, João Batista Carvalho. Monitoria acadêmica: espaço de formação. In: SANTOS, Mirza Medeiros dos; LINS, Nostradamos de Medeiros (Org.). A monitoria como espaço de iniciação à docência: possibilidades e trajetórias, Natal: EDUFRN, p. 45-58, 2007.

RAMALHO, Betânia Leite; NÚNEZ, Isauro Beltran (Org.). Formação de professores, Natal: EDUFRN, 1998.

SANTOS, Mirza Medeiros de; LINS, Nostradamos de Medeiros (Org.). A monitoria como espaço de iniciação à docência: possibilidades e trajetórias, Natal: EDUFRN, 2007.

TAVARES, J. Formação e inovação no Ensino Superior. Porto: Porto Editora, 2003.

\title{
THE RELEVANCE OF TEACHER'S ASSISTANT PROGRAM FOR CEFET'S CAMPUS ARAXÁ TEACHER'S ASSISTANTS AND STUDENTS
}

\begin{abstract}
Teacher's Assistent program is extremely important in academic's life, as it adds positively to your curriculum, in addition to improving your skills throughout training. It is up to the Teacher's Assistant to assist the student in their weaknesses regarding the subjects taught. Thus, this article aims to analyze how Teacher's Assistent program is used to enhance the collaborative and self-regulated learning of university students, with the purpose of proposing interventions that can improve the University's academic Teacher's Assistent program and the pedagogical experience of Teacher's Assistent, professors and students. Two Google Forms were applied to students in the courses of Industrial Automation Engineering and Mining Engineering at CEFET-Araxá. The first form aimed to understand the benefits and difficulties of the Teacher's Assistent and the other was intended mainly to listen to the students who regularly attended the tutoring. With the data obtained through the two surveys, the positive and negative points of the monitoring and what it impacted, especially in the formation of the student body, were analyzed. It was possible to observe that the Teacher's Assistent program brought improvements in their communication skills, an increase in their grades, in addition to a greater focus on studies. However, it is also possible to notice some problems that hinder the instruction of the Teacher's Assistent program, such as lack of adequate space for the program and the presence of uneven students regarding the reinforced content.
\end{abstract}

Keywords: Monitor. Teacher's Assistent position. Teacher's Assistant. Teacher's Assistent program. 